American Journal of Pharmacology and Toxicology 3 (1): 152-159, 2008

ISSN 1557-4962

(C) 2008 Science Publications

\title{
What is Xenohormesis?
}

\author{
Joseph A. Baur and David A. Sinclair \\ Department of Pathology, Paul F. Glenn Laboratories for the Biological Mechanisms of Aging, \\ Harvard Medical School, 77 Avenue Louis Pasteur, Boston, MA 02115
}

\begin{abstract}
Polyphenols such as resveratrol and quercetin, which are produced by stressed plants, activate sirtuin enzymes and extend the lifespan of fungi and animals, ostensibly by mimicking the beneficial effects of caloric restriction. This observation raises an interesting question: Why should foreign molecules that are non-nutritive and seemingly unrelated to any endogenous molecule modulate the same biochemical pathways that mediate the response to an energy deficit? A possible explanation is that the sirtuin enzymes have evolved to respond to plant stress molecules as indicators of an impending deterioration of the environment. This idea has become known as the Xenohormesis Hypothesis, the name stemming from a combination of the prefix xeno-(for stranger) with hormesis (a protective response induced by mild stress). Here we review the evidence for xenohormesis in a broader context, taking into account the diverse spectrum of phytochemicals to which animals are exposed. We also consider alternative hypotheses that may explain some of the beneficial effects of plant-based foods. We suggest that xenohormesis, defined as an adaptive response in the physiology of an organism to molecular cues that are neither nutritive nor direct stressors, most likely occurs at some level. Whether this can fully or partially account for the beneficial effects of resveratrol and other phytochemicals remains to be seen. However, there is already sufficient cause to re-evaluate the relationship between complex organisms, including humans and their food.
\end{abstract}

Key words: Hormesis, phytochemical, plant, resveratrol, polyphenol

\section{INTRODUCTION}

It has long been appreciated that diets rich in fruits and vegetables promote health, reduce the risk of both cancer $^{[1]}$ and cardiovascular disease ${ }^{[2]}$ and correlate with increased longevity ${ }^{[3-5]}$. This has typically been ascribed to the antioxidant properties of plant-derived foods, or the type or ratio of nutrients being absorbed $^{[6-8]}$. At the molecular level, however, it has become clear that many phytochemicals exert their effects by directly interacting with and modulating specific enzymes or receptors ${ }^{[9-12]}$. In one recent example, resveratrol, a small polyphenol found in grapes and wine, was identified in a screen for compounds that mimic the effects of Caloric Restriction (CR) by activating the sirtuin enzyme SIRT $1^{[13]}$. The sirtuins are a family of $\mathrm{NAD}^{+}$-dependent deacetylases that are conserved from yeast to humans and have been proposed to mediate lifespan extension by CR in lower organisms ${ }^{[14]}$. Consistent with this, resveratrol was found to extend the lifespan of wild type yeast, but not that of mutant strains lacking the yeast sirtuin, Sir $2^{[13]}$. It seems, therefore, that the beneficial effect of resveratrol on yeast cells is not due to a general property such as antioxidant capacity or nutritive value, but rather a very specific manipulation of a conserved signaling pathway.

It was this observation and the apparent absence of an endogenous small-molecule activator of Sir2/SIRT1, that prompted the suggestion of an alternative way by which plants might influence organisms: evolutionarily conserved survival pathways might respond to chemical cues in the environment or the food supply. To a yeast cell growing on a grape, an increase in the concentration of resveratrol, which is induced in the plant after injury or infection ${ }^{[15]}$, might be a useful indicator that the food supply is about to become limiting. Therefore the yeast cell might gain a selective advantage by responding to resveratrol in the same way it would to an actual deficit of calories, i.e. activation of sirtuin enzymes (Fig. 1). This example summarizes what has become known as the Xenohormesis Hypothesis ${ }^{[16]}$.

An important question is whether xenohormesis can reasonably be postulated to explain some of the beneficial effects of phytochemicals in higher organisms.

Corresponding Author: David A. Sinclair, Department of Pathology, Harvard Medical School, 77 Avenue Louis Pasteur, NRB 931B, Boston, MA 02115 


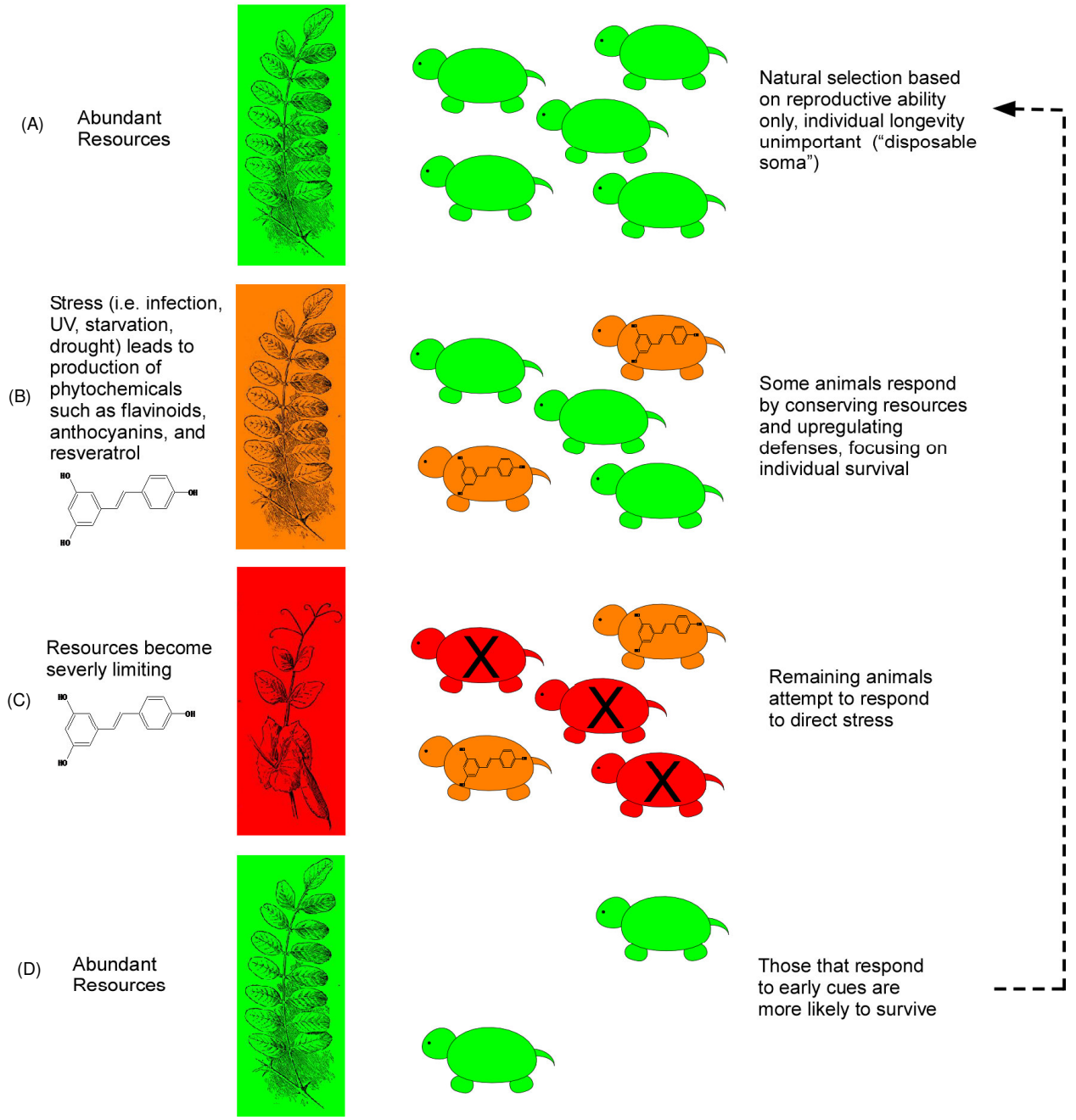

Fig. 1: A possible mechanism for the evolution of xenohormesis. (A): Under conditions where resources are not limiting, evolution favors those organisms that can reach sexual maturity and reproduce rapidly. Focusing additional effort and resources on somatic maintenance does not confer any advantage and may detract from growth and reproduction. (B): Plants experiencing mild stress synthesize a variety of phytochemicals such as resveratrol, possibly to activate their own cell defenses. Animals that can detect these molecules in their diet adjust their metabolism and boost cellular defenses in anticipation of adverse conditions. (C): Animals that responded early to cues in their diet are more likely to survive when conditions deteriorate. Those that failed to increase defenses early are more likely to die out. (D): When resources become available again the population has become enriched for animals that responded to phytochemicals. Key: : Unstressed; $\square$ : Mildly stressed; : Severely stressed

For many different molecules, including resveratrol, beneficial effects on health have been demonstrated and putatively associated with specific molecular targets ${ }^{[9-12]}$. However in many cases, the therapeutic dose of a single polyphenol is higher than what could be obtained from dietary sources alone ${ }^{[17]}$, making it hard to see how the molecules could serve as signals within the diet. Moreover, the specific profile of molecules present in the diet varies wildly depending on the plants being consumed and the stress level within those plants ${ }^{[18]}$, significantly decreasing the predictive value of any single molecule in determining future conditions.

Instead, we suggest that responses may have evolved to detect not just a single molecule in the diet but more general parameters such as the polyphenol content of the diet, common structural elements among 
inducible molecules, or a change in the substitution or oligomerization state of various phytochemicals. Although more than 8000 distinct polyphenols have been described ${ }^{[19]}$, similarities between large groups exist. For example, flavonoids, a broad class of polyphenols based on a common backbone, account for much of the diversity in the polyphenol composition of plant foods ${ }^{[18,20,21]}$ and are structurally related to other classes such as stilbenes (to which resveratrol belongs). These ubiquitous compounds are responsible for the bright colorations of many fruits and vegetables and are important mediators of stress-resistance. It is thought that flavonoids may have originated as signaling molecules $^{[22]}$, however their subsequent involvement in plant evolution has been quite complex. In addition to finding roles in protecting plants from UV irradiation, oxidative stress, toxic metals and fungal infections, flavonoids are able to attract symbiotic bacteria, suppress the growth of competing plants, selectively poison insects and deter herbivores ${ }^{[18,20,21]}$.

Despite the complexity of their structures and roles, there are some common elements to the behavior of flavonoids in plants. A large proportion are phytoalexins, joining with other polyphenols, terpenoids, alkaloids and other molecules in accumulating following UV irradiation, fungal infection, injury, possibly drought and other stresses ${ }^{[18]}$. Since flavonoids contribute the bulk of the estimated $1 \mathrm{~g}$ or $\sim 14 \mathrm{mg} \mathrm{kg} \mathrm{kay}^{-1}$ of dietary polyphenol intake $^{[17,21]}$ and many of these molecules are highly induced in stressed plants (for example, total flavonoids exuded from the roots of maize can be enhanced at least 15 -fold ${ }^{[23]}$ and the resveratrol content of various grape varieties can be increased 3.4-2315-fold ${ }^{[24]}$ compared to unstressed controls), total phytoalexin accumulation could well provide a reliable cue of a dwindling food supply.

Total dietary polyphenol intake $\left(\sim 14 \mathrm{mg} \mathrm{kg}^{-1}\right.$ day $\left.^{-1}\right)$ is well within the range for which beneficial effects have been observed using individual molecules, even before considering that our ancestors likely consumed a much more plant-rich diet during our evolution ${ }^{[25]}$. In addition, polyphenols appear to act synergistically, rather than additively in some cases and bioavailability may be enhanced depending on the combination of molecules present in the $\operatorname{diet}^{[12,26,27]}$. Non-polyphenol classes of phytoalexins (stress-induced phytochemicals) may also contribute to overall changes in the phytochemical profile of the $\operatorname{diet}^{[28]}$. In short, it is conceivable that dietary phytochemicals could replicate the beneficial results observed using pharmacological doses, if broad groups or classes act in an additive or synergistic manner.
In support of the idea that large classes of molecules could have cumulative effects, the original study that identified resveratrol as a sirtuin activator also identified 10 flavonoids and 6 other structurally related polyphenols possessing the same activity ${ }^{[13]}$. Moreover, among compounds that have been studied in relative detail, there is already a large body of evidence indicating similar effects in vitro and in vivo ${ }^{[9,11,29]}$. Resveratrol and curcumin, for example, bear little structural resemblance to one another yet both show potent anti-cancer and anti-inflammatory effects, block angiogenesis, activate glutathione-S-transferases, inhibit cyclooxygenase, possess intrinsic antioxidant capacity, induce apoptosis in tumor cells and are rapidly metabolized ${ }^{[12,30]}$. Futhermore, while the discovery of multiple direct targets for various phytochemicals has been difficult to explain in terms of other models, it is predicted by xenohormesis. This point is underscored by several examples of complimentary actions of resveratrol including the suppression of inflammation through inhibition of IL-1 $\beta$ transcription ${ }^{[31]}$, cyclooxygenase and $\mathrm{NF \kappa B}^{[12]}$ (Fig. 2). The inhibition of NF- $\mathrm{KB}$ appears to be due to both inhibition of the upstream activator $\mathrm{PKC}^{[32]}$ and activation of the inhibitor SIRT ${ }^{[33]}$. The inhibition of cyclooxygenase appears to be due to both direct binding $^{[34]}$ and transcriptional repression ${ }^{[35]}$. Cyclooxygenase is also a target of aspirin ${ }^{[36]}$, which was derived from another stress-induced phytochemical, salicylic acid. Although salicylic acid contains a phenol ring, it is structurally distinct from resveratrol and other polyphenols. It will be interesting, as more data appear, to see if the picture that emerges is one where many different plant stress molecules provoke a common series of responses, or if these examples will prove rare exceptions.

If the beneficial effects of dietary phytochemicals are to be explained as adaptive responses, two conditions must have been met during their evolution. First, phytochemical changes in the diet must have been sufficiently predictive of future environmental conditions. Second, there must have been an appropriate physiological adaptation that conferred a survival and/or reproductive advantage. There is considerable evidence that, for many organisms, both of these conditions are met. Perhaps the most important environmental change to which animals are required to adapt is the availability of food. As calories become limiting, mammals conserve energy by increasing the efficiency of metabolism, decreasing heat production, slowing growth and delaying reproduction, while enhancing maintenance and repair mechanisms. 


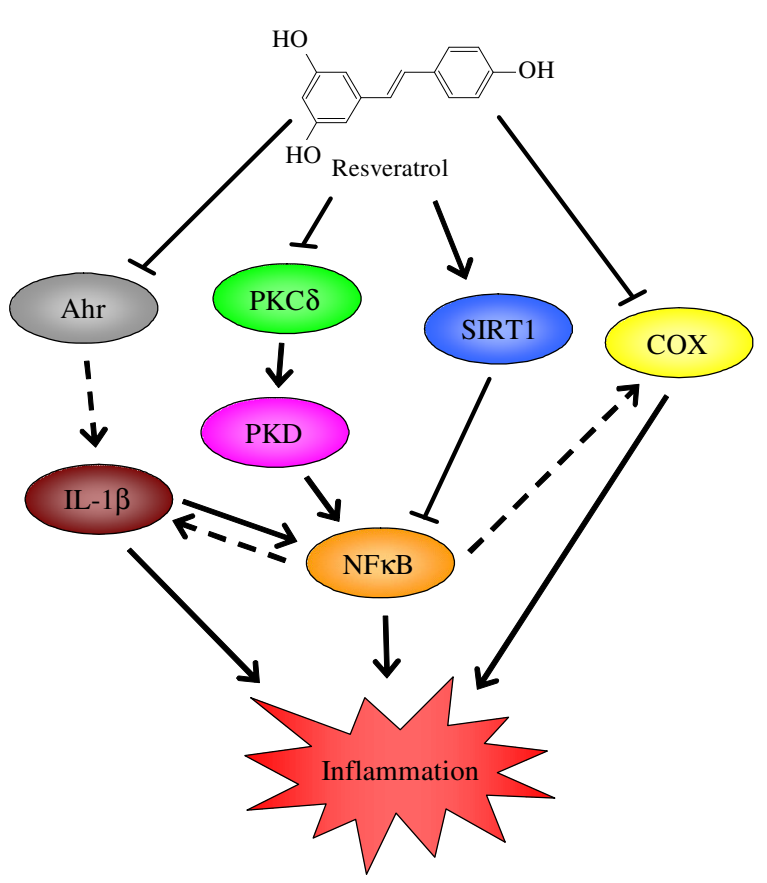

Fig. 2: Some complimentary anti-inflammatory effects of resveratrol. The four lines extending from resveratrol show direct effects. Additional antiinflammatory mechanisms, including but not limited to intrinsic antioxidant capacity and induction of antioxidant enzymes are also likely to be important. Dashed lines show transcriptional effects. Ahr-aryl: Hydrocarbon receptor; IL-1 $\beta$ : Interleukin 1 beta; PKC : Protein kinase $\mathrm{C}$ delta; PKD: Protein kinase D; NFKB: Nuclear factor kappa B; SIRT1: Sirtuin 1; COX: Cyclooxygenase

These changes are thought to improve reproductive fitness by increasing the probability of survival and subsequent reproduction in more plentiful times ${ }^{[37,38]}$. In fact caloric restriction is the most robust and reproducible regimen known to extend lifespan and improve health in mammals ${ }^{[39,40]}$. Since beneficial adaptations seems to be possible, we would predict the emergence (or continued maintenance) of a xenohormetic response to depend primarily on whether changes in the phytochemical makeup of the diet are sufficiently predictive of the availability of calories.

As we have discussed, the total level of dietary polyphenols is in the range employed for studies of single molecules ${ }^{[17,21]}$ and changes in the stress levels of plants being consumed can have a profound impact on their phytochemical makeup ${ }^{[23,24]}$. An additional mechanism by which animals could be exposed to an increased concentration of dietary polyphenols or other phytochemicals is through a change in the type of plant being consumed. Bark, for example, is a rich source of polyphenols ${ }^{[11,42]}$ but is unlikely to be selected until other food choices have been exhausted. In fact, many polyphenols appear to play specific roles in making plants less palatable to deter herbivores and insect pests $^{[20]}$, so it is possible that higher polyphenol content is a general property of less desirable plant foods. Factoring in a preference for meat, when available, would serve to strengthen the trend. In this way, increasing polyphenol content in the diet could indicate that food supplies are becoming limiting independently from any changes in the compositions of the plants themselves. One could imagine that in many cases, such as the approach of winter, food selection and plant stress-related increases in phytoalexin content would work cooperatively. Responding to seasonal change has sufficient adaptive value that animals have evolved to use almost every cue available, including day length ${ }^{[43]}$, ambient temperature ${ }^{[44,45]}$, food quality ${ }^{[46]}$, rainfall ${ }^{[47]}$ and even chemical changes in the urine of other individuals $^{[48]}$, so it would perhaps not be surprising that if a dietary phytochemical cue were available, it would be used as well.

In microbiology, the idea that molecules produced by one species can be used as cues by another species has been around for a long time. Molecules produced by various bacterial species to regulate population density, a phenomenon known as quorum sensing, have been shown to induce responses in distantly related species that do not produce similar molecules and in eukaryotic cells ${ }^{[49]}$. Certain pathogenic species of bacteria and fungi become virulent upon sensing the presence of particular flavonoids indicative of their preferred hosts ${ }^{[20]}$. But could mammals have evolved or maintained a system capable of making sense of the panoply of phytochemicals passing through our diets?

Since the bioavailability of many plant molecules appears to be limited by their rapid metabolism, a mechanism for cooperative action between molecules may be as simple as tying up the same metabolic enzymes $^{[50]}$. Alternatively, target enzymes may have evolved to tolerate some variability in structure, or even to respond synergistically to a combination of molecules. One case where mammals have evolved the ability to induce a highly coordinated response to a wide array of structurally diverse compounds is in the control of Phase I (oxidation, reduction and hydrolysis enzymes) and II (conjugating and antioxidant enzymes) responses to foreign molecules by the aryl hydrocarbon receptor (Ahr) and $\mathrm{Nrf}^{[51]}$. Ahr directly binds small molecules with broad specificity, while $\mathrm{Nrf} 2$ is 
activated by many different reactive oxygen species and electrophiles. The dynamic induction of detoxifying and antioxidant enzymes mediated by these two transcription factors is thought to limit exposure to potentially harmful foreign molecules. Xenobiotic metabolizing enzymes, particularly Phase II enzymes, may play an important role in hormetic effects. In fact, a number of carcinogens have been suggested to protect against the formation of neoplastic lesions when given at very low concentrations ${ }^{[52]}$. Although the Ahr/Nrf2 system clearly evolved for reasons that are unrelated to xenohormesis, its existence illustrates the plausibility of sensing structurally diverse molecules. It is also interesting to ask whether Ahr and/or Nrf2 might have been at least partially co-opted by xenohormesis over time. Perhaps this is related to the unexplained observation that in addition to genes involved in xenobiotic metabolism, Xenobiotic Response Elements (XREs) influence the expression of a number of genes involved in proliferation and differentiation ${ }^{[51]}$.

\section{ALTERNATIVE POSSIBILITIES}

It has been suggested that a seemingly coordinated beneficial response to a phytochemical such as resveratrol could reflect mimicry of an endogenous signaling molecule ${ }^{[13]}$. Plants and animals retain a surprising degree of similarity in certain signaling pathways ${ }^{[53]}$. Many neurotransmitters, for example, including acetylcholine, histamine, serotonin glutamate and GABA, are made by plants ${ }^{[54]}$, bolstering the hypothesis that similar signaling molecules might exist in very different species. Indeed, resveratrol has been found to interact with the estrogen receptor in some cases $^{[55]}$ and several other polyphenols have been established as bona fide estrogen mimics ${ }^{[56]}$. Recently, resveratrol was shown to have beneficial effects on bone health through an estrogen receptor-dependent mechanism $^{[57]}$. However, for the majority of beneficial effects attributed to polyphenols, no connection to estrogen signaling has been established and many of the polyphenols that display impressive health benefits are not known to affect estrogen signaling. No endogenous molecules similar to plant polyphenols have been identified in mammals and the sequencing of a number of genomes now allows us to conclude with reasonable certainty that the necessary biosynthetic pathways for polyphenols are absent. We do not, however, rule out the intriguing possibility that there are nonpolyphenolic endogenous modulators waiting to be discovered. Importantly, if they are discovered, their existence would not rule out Xenohormesis.
Another possibility is that the common denominator among phytochemicals is their antioxidant capacity. Certainly antioxidant action has historically been considered the major mechanism contributing to the beneficial effects of a diet rich in fruits and vegetables. Intensive study of antioxidant vitamins in recent years has failed to produce convincing evidence of either disease prevention, or lifespan extension, leading some to question this premise ${ }^{[7,58]}$. However polyphenols, not the more familiar vitamins, are the major source of antioxidants in natural foods and may have important properties, such as the ability to accumulate in specific subcellular compartments, that vitamin antioxidants lack $^{[6]}$. Interpreting antioxidant effects of polyphenols in vivo is quite problematic since they tend to induce expression of antioxidant enzymes. Resveratrol, for example, induces expression of catalase and quinone reductase 1 in the cardiac tissue of guinea pigs $^{[59]}$. When decreases in markers of oxidative stress are observed, therefore, it is not always clear whether this represents direct antioxidant activity, or an adaptive response on the part of the host. One argument for the latter is that, as recently pointed out by Halliwell ${ }^{[6]}$, unconjugated polyphenols rarely exceed the low uM range in plasma, while total antioxidant capacity is often in the range of $1 \mathrm{mM}$. We do not exclude a role for direct antioxidant effects in the benefits of polyphenols, but do feel that this explanation fails to adequately account for the many, seemingly coordinated effects.

A third possibility is that beneficial phytochemicals provoke an adaptive response, but by acting as or mimicking direct stressors. This would qualify as an example of hormesis (a net beneficial effect when stress-response pathways are activated by a mild stress). In contrast, xenohormesis postulates that these molecules serve as benign cues in the diet and that animals can again a selective advantage by evolving mechanisms to detect them. The most obvious way phytochemicals might be perceived as direct stressors and thereby induce a hormetic response, is through the Phase I and II xenobiotic responses mentioned above $^{[60]}$. Since the Phase II response in particular involves the induction of antioxidant and other protective enzymes in order to limit damage caused by foreign molecules, chronic stimulation of this pathway might be expected to have beneficial consequences. Indeed, a number of beneficial phytochemicals, including resveratrol and curcumin, as well as more complex extracts, have been shown to induce Phase II enzymes and have other effects on xenobiotic metabolism $^{[30,61-63]}$ and this most likely plays a role in their effects in vivo. However, this explanation cannot 
readily account for many of the specific targets that have been elucidated for these molecules. Enzymes such as SIRT1, cyclooxygenase, PKC and others bind directly to resveratrol and are thought to be key players in mediating its beneficial effects. Moreover, given sufficient selective pressure to produce xenohormetic responses, integration of these adaptations into the existing Phase I and II responses would likely be the path of least resistance from an evolutionary point of view. Therefore a canonical xenobiotic stress response probably plays a role in the physiological changes induced by phytochemicals, but is unlikely to be the whole explanation.

\section{CONCLUSION}

There is much that remains to be understood about the complex relationship between animals and the plants they consume. Throughout evolution, the flux of various phytochemicals in food sources would have provided a wealth of information to an animal capable of deciphering the signals. We argue that given the existence of such signals and selective pressure to adapt to environmental changes, xenohormesis likely occurs at some level. To what extent this phenomenon might contribute to the beneficial effects of specific phytochemicals, or fruits and vegetables in general, remains to be seen. If we are correct, then stressed plants may contain a host of potentially therapeutic molecules that have yet to be recognized. A further consideration is the timing of therapeutic effects. Direct stresses may induce protective hormetic responses early in life, but have diminished or even detrimental effects later in life. Similarly, there may be an optimal therapeutic window in which a protective xenohormetic response can be established. Elucidating the mechanism by which plant foods benefit human health has enormous potential to improve quality of life and prevent or treat major diseases. Progress toward this goal, including further testing of the Xenohormesis hypothesis, will be an important challenge in the coming years.

Note added in proof: Since the submission of this review, two studies have shown that resveratrol treatment broadly mimics the transcriptional effects of caloric restriction (Barger et al., PLoS ONE, 3(6): e2264, 2008, Pearson et al., Cell Metabolism, in press, 2008), strengthening the case that both might act through a common mechanism.

\section{REFERENCES}

1. Block, G., B. Patterson and A. Subar, 1992. Fruit, vegetables and cancer prevention: A review of the epidemiological evidence. Nutr. Cancer, 18: 1-29.

2. Bazzano, L.A., M.K. Serdula and S. Liu, 2003. Dietary intake of fruits and vegetables and risk of cardiovascular disease. Curr. Atheroscler Rep., 5: 492-499.

3. Suzuki, M., B.J. Wilcox and C.D. Wilcox, 2001. Implications from and for food cultures for cardiovascular disease: Longevity. Asia Pac J. Clin. Nutr., 10: 165-171.

4. Trichopoulou, A. and E. Vasilopoulou, 2000. Mediterranean diet and longevity. Br. J. Nutr., 84: S205-S209.

5. Singh, P.N., J. Sabate and G.E. Fraser, 2003. Does low meat consumption increase life expectancy in humans? Am. J. Clin. Nutr., 78: 526S-532S.

6. Halliwell, B., 2007. Dietary polyphenols: Good, bad, or indifferent for your health? Cardiovasc. Res., 73: 341-347.

7. Lotito, S. B. and B. Frei, 2006. Consumption of flavonoid-rich foods and increased plasma antioxidant capacity in humans: Cause, consequence, or epiphenomenon? Free Radic. Biol. Med., 41: 1727-1746.

8. Liu, R.H., 2003. Health benefits of fruit and vegetables are from additive and synergistic combinations of phytochemicals. Am. J. Clin. Nutr., 78: 517S-520S.

9. Williams, R.J., J.P. Spencer and C. Rice-Evans, 2004. Flavonoids: Antioxidants or signalling molecules? Free Radic. Biol. Med., 36: 838-849.

10. Thangapazham, R.L., A. Sharma and R.K. Maheshwari, 2006. Multiple molecular targets in cancer chemoprevention by curcumin. Aaps. J., 8: E443-W4549.

11. Singh, R.P., S. Dhanalakshmi and R. Agarwal, 2002. Phytochemicals as cell cycle modulators-a less toxic approach in halting human cancers. Cell Cycle, 1: 156-161.

12. Baur, J.A. and D.A. Sinclair, 2006. Therapeutic potential of resveratrol: The in vivo evidence. Nat. Rev. Drug Discov., 5: 493-506.

13. Howitz, K.T., K.J. Bitterman, H.Y. Cohen, D.W. Lamming, S. Lavu, J.G. Wood, R.E. Zipkin, P. Chung, A. Kisielewski, L.L. Zhang, B. Scherer and D.A. Sinclair, 2003. Small molecule activators of sirtuins extend Saccharomyces cerevisiae lifespan. Nature, 425: 191-196. 
14. Guarente, L., 2005. Calorie restriction and SIR2 genes-towards a mechanism. Mech. Ageing Dev., 126: 923-928.

15. Langcake, P. and R.J. Pryce, 1976. The production of resveratrol by Vitis vinifera and other members of the Vitaceae as a response to infection or injury. Physiol. Plant Pathol., 9: 77-86.

16. Lamming, D.W., J.G. Wood and D.A. Sinclair, 2004. Small molecules that regulate lifespan: Evidence for xenohormesis. Mol. Microbiol., 53: 1003-1009.

17. Scalbert, A., I.T. Johnson and M. Saltmarsh, 2005. Polyphenols: Antioxidants and beyond. Am. J. Clin. Nutr., 81: 215S-217S.

18. Winkel-Shirley, B., 2002. Biosynthesis of flavonoids and effects of stress. Curr. Opin. Plant Biol., 5: 218-23.

19. Bravo, L., 1998. Polyphenols: Chemistry, dietary sources, metabolism and nutritional significance. Nutr. Rev., 56: 317-333.

20. Treutter, D., 2005. Significance of flavonoids in plant resistance and enhancement of their biosynthesis. Plant Biol. (Stuttg), 7: 581-591.

21. Aherne, S.A. and N.M. O'Brien, 2002. Dietary flavonols: Chemistry, food content and metabolism. Nutrition, 18: 75-81.

22. Stafford, H.A., 1991. Flavonoid evolution: An enzymic approach. Plant Physiol., 96: 680-685.

23. Kidd, P.S., M. Llugany, C. Poschenrieder, B. Gunse and J. Barcelo, 2001. The role of root exudates in aluminium resistance and silicon-induced amelioration of aluminium toxicity in three varieties of maize (Zea mays L.). J. Exp. Bot., 52: 1339-1352.

24. Cantos, E., J.C. Espin and F.A. Tomas-Barberan, 2002. Postharvest stilbene-enrichment of red and white table grape varieties using UV-C irradiation pulses. J. Agric. Food Chem., 50: 6322-6329.

25. Milton, K., 2003. Micronutrient intakes of wild primates: Are humans different? Comp. Biochem. Physiol. A. Mol. Integr. Physiol., 136: 47-59.

26. HemaIswarya, S. and M. Doble, 2006. Potential synergism of natural products in the treatment of cancer. Phytother. Res., 20: 239-249.

27. De Santi, C., A. Pietrabissa, R. Spisni, F. Mosca and G.M. Pacifici, 2000. Sulphation of resveratrol, a natural product present in grapes and wine, in the human liver and duodenum. Xenobiotica, 30: 609-617.

28. Hammerschmidt, R., 1999. PHYTOALEXINS: What Have We Learned After 60 Years? Annu. Rev. Phytopathol., 37: 285-306.
29. Zheng, J. and V.D. Ramirez, 2000. Inhibition of mitochondrial proton F0F1-ATPase/ATP synthase by polyphenolic phytochemicals. Br. J. Pharmacol., 130: 1115-1123.

30. Sharma, R.A., A.J. Gescher and W.P. Steward, 2005. Curcumin: The story so far. Eur. J. Cancer, 41: 1955-1968.

31. Casper, R.F., M. Quesne, I.M. Rogers, T. Shirota, A. Jolivet, E. Milgrom and J.F. Savouret, 1999. Resveratrol has antagonist activity on the aryl hydrocarbon receptor: Implications for prevention of dioxin toxicity. Mol. Pharmacol., 56: 784-90.

32. Storz, P., H. Doppler and A. Toker, 2004. Activation loop phosphorylation controls protein kinase D-dependent activation of nuclear factor kappaB. Mol. Pharmacol., 66: 870-879.

33. Yeung, F., J.E. Hoberg, C.S. Ramsey, M.D. Keller, D.R. Jones, R.A. Frye and M.W. Mayo, 2004. Modulation of NF-kappaB-dependent transcription and cell survival by the SIRT1 deacetylase. EMBO J., 23: 2369-2380.

34. Jang, M., L. Cai, G.O. Udeani, K.V. Slowing, C.F. Thomas, C.W. Beecher, H.H. Fong, N.R. Farnsworth, A.D. Kinghorn, R.G. Mehta, R.C. Moon and J.M. Pezzuto, 1997. Cancer chemopreventive activity of resveratrol, a natural product derived from grapes. Science, 275: 218-220.

35. Subbaramaiah, K., W.J. Chung, P. Michaluart, N. Telang, T. Tanabe, H. Inoue, M. Jang, J.M. Pezzuto and A.J. Dannenberg, 1998. Resveratrol inhibits cyclooxygenase-2 transcription and activity in phorbol ester-treated human mammary epithelial cells. J. Biol. Chem., 273: 21875-21882.

36. Vane, J.R., 1971. Inhibition of prostaglandin synthesis as a mechanism of action for aspirin-like drugs. Nat. New Biol., 231: 232-235.

37. Harrison, D.E. and J.R. Archer, 1989. Natural selection for extended longevity from food restriction. Growth Dev. Aging, 53: 3.

38. Holliday, R., 1989. Food, reproduction and longevity: Is the extended lifespan of calorierestricted animals an evolutionary adaptation? Bioessays, 10: 125-127.

39. McCay, C.M. and M.F. Crowell, 1934. Prolonging the lifespan. Sci. Monthly, 39: 405-414.

40. Roth, G.S., M.A. Lane and D.K. Ingram, 2005. Caloric restriction mimetics: The next phase. Ann. N.Y. Acad. Sci., 1057: 365-371.

41. Nahrstedt, A., M. Schmidt, R. Jaggi, J. Metz and M.T. Khayyal, 2007. Willow bark extract: The contribution of polyphenols to the overall effect. Wien. Med. Wochenschr., 157: 348-351. 
42. Devaraj, S., S. Vega-Lopez, N. Kaul, F. Schonlau, P. Rohdewald and I. Jialal, 2002. Supplementation with a pine bark extract rich in polyphenols increases plasma antioxidant capacity and alters the plasma lipoprotein profile. Lipids, 37: 931-934.

43. Haim, A., U. Shanas, S. Zubidad Ael and M. Scantelbury, 2005. Seasonality and seasons out of time-the thermoregulatory effects of light interference. Chronobiol. Int., 22: 59-66.

44. Johnston, I.A. and G.K. Temple, 2002. Thermal plasticity of skeletal muscle phenotype in ectothermic vertebrates and its significance for locomotory behaviour. J. Exp. Biol., 205: 2305-2322.

45. Kobayashi, M., T. Ito, S. Ishii and M. Wada, 2004. Seasonal change in luteinizing hormone subunit mRNA in Japanese quail and effects of short daylength and low temperature. Gen. Comput. Endocrinol., 139: 38-47.

46. Geiser, F., G.J. Kenagy and J.C. Wingfield, 1997. Dietary cholesterol enhances torpor in a rodent hibernator. J. Comput. Physiol. [B], 167: 416-422.

47. Kelly, J.P., N. Warnock, G.W. Page and W.W. Weathers, 2002. Effects of weather on daily body mass regulation in wintering dunlin. J. Exp. Biol., 205: 109-120.

48. Heth, G., E. Nevo and J. Todrank, 1996. Seasonal changes in urinary odors and in responses to them by blind subterranean mole rats. Physiol. Behav., 60: 963-968.

49. Diggle, S.P., A. Gardner, S.A. West and A.S. Griffin, 2007. Evolutionary theory of bacterial quorum sensing: When is a signal not a signal? Philos. Trans. R. Soc. Lond. B. Biol. Sci., 362: 1241-1249.

50. Silberberg, M., C. Morand, T. Mathevon, C. Besson, C. Manach, A. Scalbert and C. Remesy, 2006. The bioavailability of polyphenols is highly governed by the capacity of the intestine and of the liver to secrete conjugated metabolites. Eur. J. Nutr., 45: $88-96$.

51. Kohle, C. and K.W. Bock, 2007. Coordinate regulation of Phase I and II xenobiotic metabolisms by the Ah receptor and Nrf2. Biochem. Pharmacol., 73: 1853-1862.

52. Fukushima, S., A. Kinoshita, R. Puatanachokchai, M. Kushida, H. Wanibuchi and K. Morimura, 2005. Hormesis and dose-response-mediated mechanisms in carcinogenesis: Evidence for a threshold in carcinogenicity of non-genotoxic carcinogens. Carcinogenesis, 26: 1835-1845.

53. Schultz, J.C., 2002. Shared signals and the potential for phylogenetic espionage between plants and animals. Integ. Comput. Biol., 42: 454-462.
54. Roshchina, V.V., 2001. Neurotransmitters in Plant Life. 1st Edn., Science Publishers, Enfield, NH.

55. Gehm, B.D., J.M. McAndrews, P.Y. Chien and J.L. Jameson, 1997. Resveratrol, a polyphenolic compound found in grapes and wine, is an agonist for the estrogen receptor. Proc. Natl. Acad. Sci. USA., 94: 14138-14143.

56. Fletcher, R.J., 2003. Food sources of phytooestrogens and their precursors in Europe. Br. J. Nutr., 89: S39-S43.

57. Su, J.L., C.Y. Yang, M. Zhao, M.L. Kuo and M.L. Yen, 2007. Forkhead proteins are critical for bone morphogenetic protein-2 regulation and antitumor activity of resveratrol. J . Biol. Chem., 282: 19385-19398.

58. Kamel, N.S., J. Gammack, O. Cepeda and J.H. Flaherty, 2006. Antioxidants and hormones as antiaging therapies: High hopes, disappointing results. Cleve. Clin. J. Med., 73: 1049-1056, 1058.

59. Floreani, M., E. Napoli, L. Quintieri and P. Palatini, 2003. Oral administration of trans-resveratrol to guinea pigs increases cardiac DT-diaphorase and catalase activities and protects isolated atria from menadione toxicity. Life Sci., 72: 2741-2750.

60. Kohle, C. and K.W. Bock, 2006. Activation of coupled Ah receptor and Nrf2 gene batteries by dietary phytochemicals in relation to chemoprevention. Biochem. Pharmacol., 72: 795-805.

61. Cao, Z. and Y. Li, 2004. Potent induction of cellular antioxidants and phase 2 enzymes by resveratrol in cardiomyocytes: Protection against oxidative and electrophilic injury. Eur. J. Pharmacol., 489: 39-48.

62. Kaga, S., L. Zhan, M. Matsumoto and N. Maulik, 2005. Resveratrol enhances neovascularization in the infarcted rat myocardium through the induction of thioredoxin-1, heme oxygenase-1 and vascular endothelial growth factor. J. Mol. Cell. Cardiol., 39: 813-822.

63. Myhrstad, M.C., H. Carlsen, L.I. Dahl, K. Ebihara, L. Glemmestad, K. Haffner, J.O. Moskaug and R. Blomhoff, 2006. Bilberry extracts induce gene expression through the electrophile response element. Nutr. Cancer, 54: 94-101. 